\title{
Investigación del desgaste de la leva anular de la bomba de alta presión DENSO HP3 para sistemas CRDI mediante transformada de Fourier y análisis de desempeño al utilizar una mezcla ecológica diésel - surfactante como combustible alternativo
}

\author{
Investigation of the wear of the annular cam of the high pressure pump DENSO HP3 \\ for CRDI systems within the use of the Fourier Transform and the analysis of the \\ performance using an ecological mixture of Diesel - surfactant as an alternative fuel \\ Germán Erazo $^{1 *} \quad$ Julieta Bassante Barberán ${ }^{1} \quad$ Santiago Díaz Vivanco ${ }^{1}$ \\ Marcelo Román ${ }^{1} \quad$ Norma Barreno $^{1} \quad$ Alexandra Corral $^{1}$ \\ Recibido 25 de marzo de 2019, aceptado 13 de marzo de 2020 \\ Received: March 25, 2019 Accepted: March 13, 2020
}

\begin{abstract}
RESUMEN
Se aplica la transformada de Fourier en el análisis del desgaste superficial de la leva anular de la bomba de alta presión DENSO HP3 al utilizar la mezcla combustible ecológica diésel - surfactante - agua al 20\%, que es una emulsión de dos sustancias tenso activas no iónicas, durante 200 horas de funcionamiento a 3000 rpm, con inspecciones cada 50 horas. El combustible ecológico reduce las emisiones, aumenta la eficiencia térmica, torque y potencia del motor de combustión interna, como demuestra el análisis experimental, la balanza de precisión detecta la reducción de 0,121 gramos en la masa de la leva, el rugosímetro digital determina la rugosidad $R a$ en la cara frontal de la leva estableciendo el 22,33\% de desgaste en relación con el perfil ideal de experimentación, para la cara posterior 40,35\% de desgaste, con el módulo didáctico generado en Matlab para el procesamiento digital de imágenes y la transformada de Fourier se compara el perfil ideal y el perfil de rugosidad de la leva anular en términos de la frecuencia de las imágenes, aplicando herramientas estadísticas como el promedio del máximo y mínimo de intensidad. El uso de la mezcla combustible diésel surfactantes agua al 20\%, en la bomba de alta presión HP3 genera desgastes prematuros en las superficies de la leva. Con el propósito de generar un aporte investigativo se incluyen la metodología y técnica de pruebas en el dinamómetro para estimar las características de desempeño fundamental del motor de combustión interna diésel con este tipo de biocombustible.
\end{abstract}

Palabras clave: Rugosidad, emulsión de combustible, CRDI, surfactantes, Fourier, procesamiento de imágenes.

\section{ABSTRACT}

The Fourier Transform is applied in the analysis of the surface wear of the annular cam of the highpressure pump DENSO HP3 using the ecological fuel mixture diesel - surfactant - water at 20\%, which is an emulsion of two tense-active non-ionic substances during 200 functioning hours at 3000 rpm with inspections performed each 50 hours. The ecological fuel reduces the emissions, enhance the thermal

\footnotetext{
1 Universidad de las Fuerzas Armadas ESPE. Dpto. Energía y Mecánica, SAEM R\&D, Dpto. Ciencias Exactas, Dpto. Electrónica. Sangolqui, Ecuador. E-mail: wgerazo@espe.edu.ec; jmbassante@espe.edu.ec; asdiaz5@espe.edu.ec; wmroman@espe.edu.ec; npbarreno@espe.edu.ec; macorral@espe.edu.ec.

* Autor de correspondencia: wgerazo@espe.edu.ec
} 
efficiency, torque, and power of the internal combustion motor; as it is shown in the experimental analysis, the precision balance it is detected the reduction of 0.121 grams of mass in the cam, with the digital roughness meter it is determined the roughness $R a$ on the frontal face of the cam, establishing a $22.23 \%$ of wear in relation with the ideal profile of experimentation, for the rear face $40.35 \%$ of wear, with the didactic module generated on Matlab for the digital image processing and the Fourier Transform compares the ideal profile and the roughness profile of the annular cam in terms of image frequency, using statistics tools such as the maximum average and the minimum o intensity. The use of the mixture of the diesel-surfactant-water fuel at $20 \%$ on the high-pressure pump HP3 generates premature wear on the cam surfaces. With the purpose of generating an investigating contribution, it is included the methodology and the technic of tests and measurements on the dynamometer for estimating the fundamental performance characteristics of the diesel internal combustion motor with this type of fuel.

Key Words: Roughness, fuel emulsion, CRDI, surfactants, Fourier, image processing.

\section{INTRODUCCIÓN}

El sistema riel común CRDI ajusta con precisión la cantidad de combustible para el motor de combustión interna, generando presiones de inyección desde 400 bar hasta 1800 bar de acuerdo a la condición de aceleración y carga [1], reduce del consumo de combustible y la emisión de gases al medio ambiente [2]. Por el efecto contaminante que producen los combustibles fósiles; se investiga sobre el uso de alternativos y ecológicos [3].

[4] Los combustibles alternos producidos a partir de materias de base renovables, permiten no solo dejar que se dependa del petróleo para obtener combustibles, sino también una alternativa amigable con el medio ambiente.

La emulsión de combustible permite la mezcla de agua $\mathrm{u}$ otra sustancia inmiscible generalmente en aceite de manera que sea lo más homogénea posible; mediante el uso de tenso activos, se evita la separación de fases. La aplicación de las emulsiones de combustible reduce la temperatura de combustión, las emisiones contaminantes y aumentan el rendimiento térmico debido a micro explosiones generadas por las partículas de agua [5]. Los valores de BHL (Balance Hidrofílico Lipofílico) de los surfactantes permiten clasificarlos de acuerdo a su mayor o menor solubilidad, sea en aceite o en agua [6].

En funcionamiento los componentes del sistema CRDI al entrar en constante contacto, generan desgastes en las superficies de sus elementos. Según [7] el proceso de desgaste, produce una pérdida de material bajo la acción de una fuerza, este desgaste conlleva a una disminución de tolerancia de las medidas de fabricación.

Investigaciones previas [8,9] detallan que los diferentes tipos de desgaste dependen de las condiciones de trabajo a las que el elemento está sometido. El desgaste mediante proyecciones microscópicas de la unión hace que las fuerzas de deslizamiento fracturen la unión, desgarrando al material de una superficie [9].

El desgaste por corrosión [10] se produce por las reacciones químicas a las que está sometida la leva anular al trabajar con el diésel - surfactante agua. La superficie de la leva anular tiene un acabo superficial de gran calidad debido a las condiciones en las que trabaja, con una composición química y ordenamiento atómico especial [11]. La rugosidad analizada establece la condición de su superficie, durabilidad y el efecto que se genera al trabajar con distintos tipos de combustibles [8].

La problemática de realizar el análisis de rugosidad de piezas mecánicas, es la detección visual de las deformaciones producidas en las superficies. La investigación es de tipo empírico, ya que se somete a los componentes del sistema CRDI a pruebas de funcionamiento con la mezcla ecológica durante 200 horas a $3000 \mathrm{rpm}$. Se realiza el procesamiento digital de imágenes, en [12] propone el método de supresión de franjas basado en la transformación de Fourier y la utilización de filtros para la detección imágenes topográficas. En [13] presenta el algoritmo para identificar espectros de la imagen digital, en [14, 15] está la guía para evaluar, analizar y comparar la eficiencia de las diferentes Transformadas Discretas 
Fraccionarias de Fourier en el tratamiento de imágenes, en este contexto a través de la interfaz gráfica en Matlab analiza y compara las imágenes de la leva anular de la bomba de alta presión en términos de la rugosidad o desgaste superficial, como base de la investigación. Se considera las fotografías tomadas a las $0,50,100,150$ y 200 horas de funcionamiento del motor de combustión, analizando el dominio de frecuencia utilizando técnicas del análisis de Fourier [16], identificando dominios de frecuencia críticos en los cuales se aplica el método de imagen multi espectral [17].

Se aplica la técnica de representación matricial a imágenes digitales, por lo tanto, el desarrollo de la aplicación consiste en binarizar los patrones de alta y baja frecuencia [18] aplicando el siguiente procedimiento:

- Adquisición de la imagen.

- Aplicar la transformada de Fourier a la imagen para interpretar la señal discreta bidimensional como una suma finita de ondas sinodales.

- Implementar filtros para identificar patrones de variación en la imagen.

- Aplicar la transformada inversa de Fourier para la reconstrucción de la imagen digital procesada.

- Aplicar técnicas estadísticas al procesamiento de imágenes con el objetivo de encontrar diferencias entre la verificación visual y el análisis digital, en este punto se implementó la técnica del análisis de las piezas mecánicas en función de su peso con el objetivo de correlacionar los resultados encontrados.

Para la validación de resultados se emplean técnicas estadísticas para cada caso mencionado.

\section{MATERIALES Y MÉTODOS}

\section{Mezcla combustible}

La mezcla combustible ecológica elaborada [19], contiene diésel Premium, agua destilada y tenso activos que estabilizan las dos sustancias a una concentración del 20\%. La Tabla 1 presenta los valores de BHL de los tenso activos, la mezcla de los emulsionantes en las cantidades indicadas permiten obtener una mezcla con un BHL4 (Balance Hidrofílico Lipofílico) que permite la combinación entre agua y aceite.

Se combina las cantidades de las sustancias para elaborar un kilogramo de mezcla [20], combinando
Tabla 1. Balance Hidrofílico Lipofílico (BHL) de tenso activos.

\begin{tabular}{|l|c|c|}
\hline \multicolumn{1}{|c|}{ Tenso activo } & BHL & Cantidad (g) \\
\hline Ácido oleico & 1 & 66,7 \\
\hline Kolliphor & 14 & 20 \\
\hline Nonil fenol de 6 moles & 10,9 & 33,3 \\
\hline
\end{tabular}

Tabla 2. Cantidades necesarias de cada sustancia.

\begin{tabular}{|l|c|c|}
\hline \multicolumn{1}{|c|}{ Sustancia } & $\begin{array}{c}\text { Cantidad } \\
(\mathbf{g})\end{array}$ & $\begin{array}{c}\text { Porcentaje } \\
(\mathbf{\%})\end{array}$ \\
\hline Ácido oleico & 66,7 & 6,67 \\
\hline Kolliphor & 33,3 & 3,33 \\
\hline Nonil fenol de 6 moles & 20 & 2 \\
\hline Agua destilada & 200 & 20 \\
\hline Diésel & 680 & 68 \\
\hline
\end{tabular}

tenso activos por separado. La Tabla 2 detalla las cantidades necesarias de cada componente.

Para preparar la mezcla combustible, se requiere el equipo de agitación Figura 1, para la correcta homogeneización a 600 rpm [20].

En la Figura 2 se presenta el agitador magnético BOECO que agita la mezcla para evitar la separación posterior.

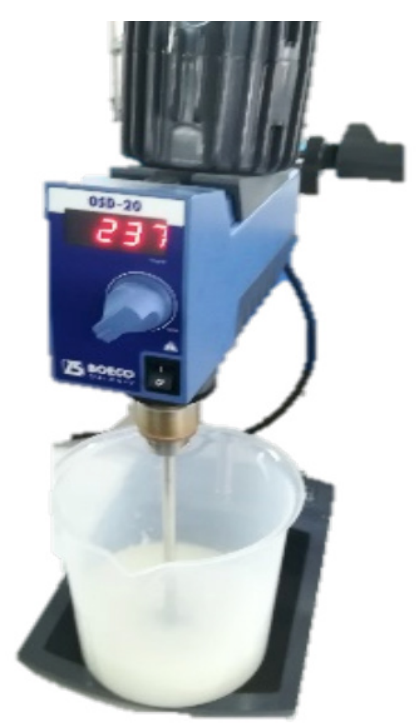

Figura 1. Agitador mecánico OSD -20 BOECO. 


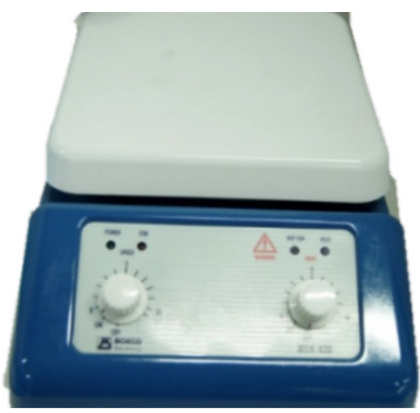

Figura 2. Agitador magnético MSH 420 BOECO.

\section{Banco de pruebas CRDI}

En la Figura 3 está el banco de pruebas de inyección diésel CRDI, en el cual se instala la bomba de alta presión HP3 [21], así como el módulo activador de inyectores.

La Tabla 3 presenta los parámetros ajustados para las pruebas en el banco.

\section{Módulo activador}

Para la activación del inyector CRDI, se utiliza el módulo de la Figura 4, dispone de la etapa de control

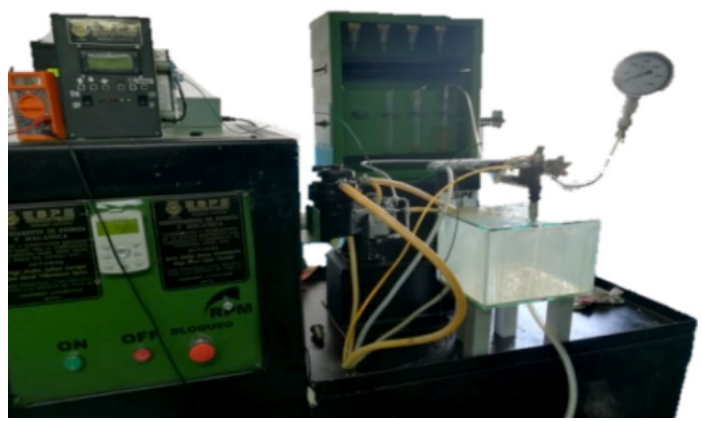

Figura 3 Banco de pruebas CRDI.

Tabla 3. Parámetros de funcionamiento del banco de pruebas CRDI.

\begin{tabular}{|l|r|c|}
\hline \multicolumn{1}{|c|}{ Parámetro } & Valor & Unidad \\
\hline Velocidad de giro & 900 & RPM \\
\hline Presión en el riel & 400 & Bar \\
\hline Período de inyección & 48 & $\mathrm{~ms}$ \\
\hline Tiempo de inyección & 1,6 & $\mathrm{~ms}$ \\
\hline Presión de alimentación & 1 & $\mathrm{Bar}$ \\
\hline Voltaje de activación & 90 & $\mathrm{~V}$ \\
\hline Amperaje de activación & 15 & $\mathrm{~A}$ \\
\hline
\end{tabular}

y de potencia para ajustar el tiempo de activación y retención; eleva el voltaje a 90 voltios y proporciona hasta 20 amperios de corriente [1], conmutando los valores necesarios para la investigación.

En la Figura 5 está la bomba de alta presión DENSO HP3, se instala en el banco de pruebas CRDI, es accionada por un motor eléctrico trifásico de 200 Voltios, con una frecuencia ajustable de 0 a $60 \mathrm{~Hz}$ [21].

\section{Medición de la superficie de la leva anular}

En la Figura 6 se presenta el procedimiento para la medición de la rugosidad de la leva anular, que asegure la calidad de datos obtenidos con el rugosímetro.

En la Figura 7 la balanza analítica ADAM, con una apreciación de 0,0001 gramos, verifica la pérdida de masa que sufre la leva al trabajar con la mezcla combustible durante cada intervalo pruebas.

En la Figura 8 el rugosímetro digital CMSRT210, establece la medida de rugosidad del desgaste en

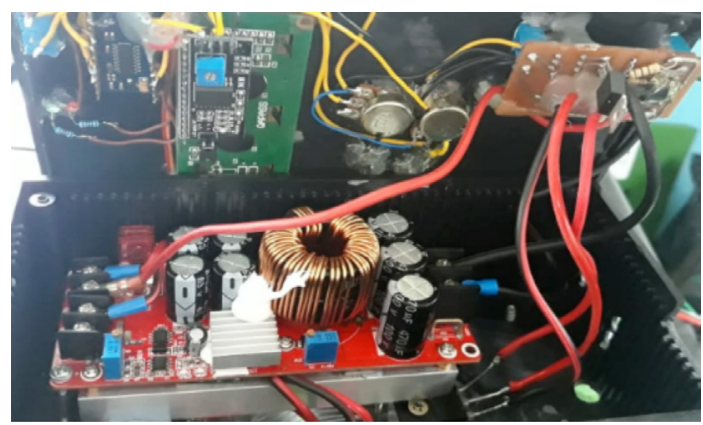

Figura 4. Módulo de activación de inyectores.

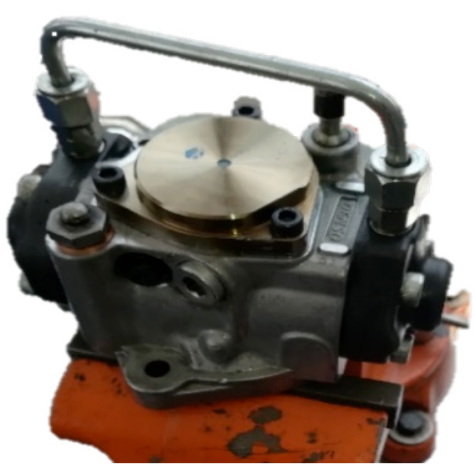

Figura 5. Bomba de alta presión HP3. 


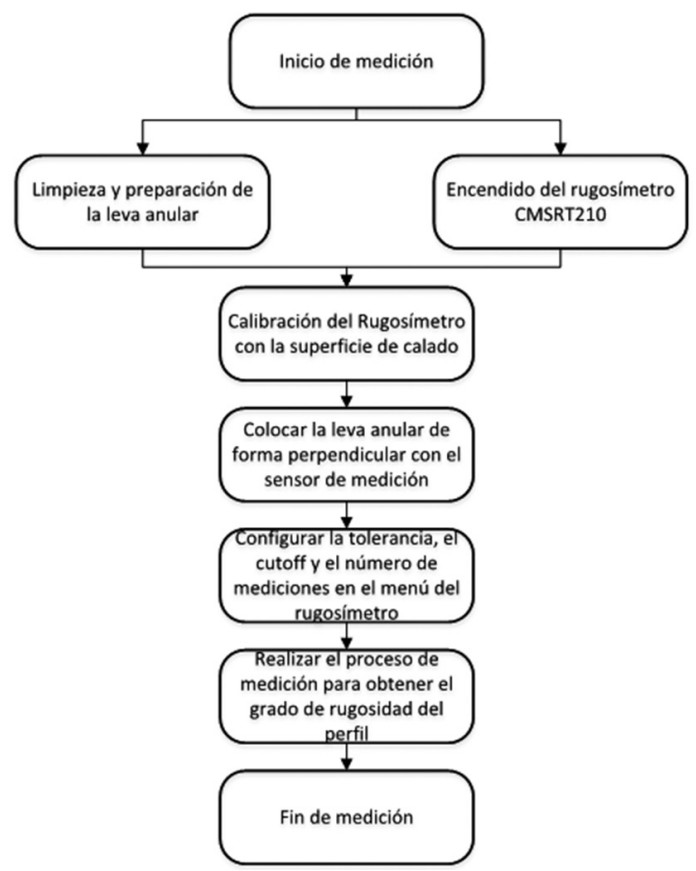

Figura 6. Procedimiento de medición de la rugosidad.

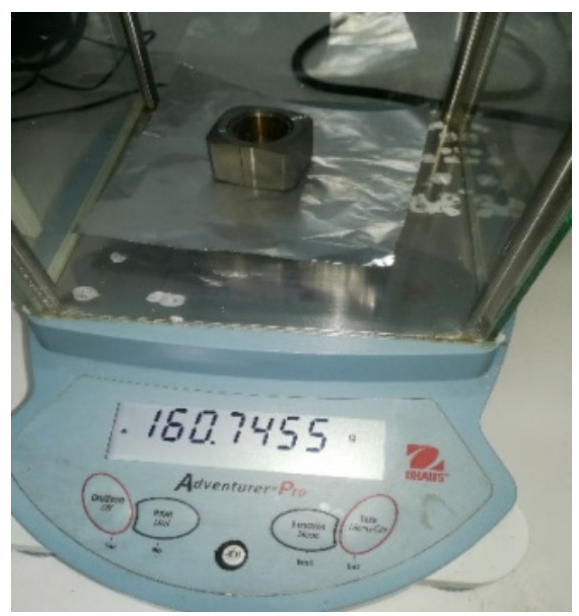

Figura 7. Pesaje de la leva anular.

la superficie de la leva anular, según [24] el valor a inspeccionar se encuentra de 0,1 a $2 \mu \mathrm{m}$, por lo que se configura el rugosímetro mediante la Tabla 4 un cut off de 0,8 mm y una longitud de medición de $4 \mathrm{~mm}$, de acuerdo a la norma DIN 4768.

Los parámetros analizados son el $R a$ y $R q$, el procedimiento consiste en realizar la inspección de la superficie de la leva anular con el sensor del

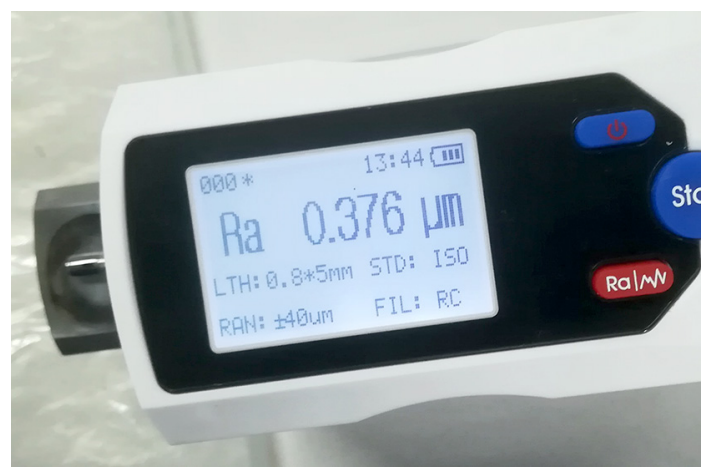

Figura 8. Rugosímetro CMSRT210.

Tabla 4. Correspondencia para la rugosidad media Ra.

\begin{tabular}{|c|r|r|r|}
\hline $\begin{array}{c}\mathrm{Ra} \\
\mu \mathrm{m}\end{array}$ & $\begin{array}{c}\lambda \mathrm{c} \\
\mathrm{Mn}\end{array}$ & $\begin{array}{c}\text { Lo } \\
\mathrm{Mm}\end{array}$ & $\begin{array}{c}\text { Lm } \\
\mathrm{mm}\end{array}$ \\
\hline Hasta 0,1 & 0,25 & 0,25 & 1,25 \\
\hline$>0,1-2$ & 0,8 & 0,8 & 4 \\
\hline$>2-10$ & 2,5 & 2,5 & 12,5 \\
\hline$>10$ & 8 & 8 & 40 \\
\hline
\end{tabular}

rugosímetro, el cual una vez calibrado, genera la lectura de los valores reales de $R a$ de la superficie.

- Parámetro de rugosidad $R a$

$$
R_{a}=\frac{1}{L} \int_{0}^{L}|f(x)| d x
$$

Donde:

- $L$ longitud de muestreo

- $\quad x$ representa la línea media entre los picos y valles de la superficie muestreada, con el eje $y$ en la dirección de la amplificación longitudinal de dicha curva de muestreo la misma que es expresada por $y=f(x)$.

En [25], la ecuación (1) es implementada en la programación en Matlab de forma discretizada para analizar las ordenadas del perfil de la fotografía.

- Parámetro de rugosidad $R q$

$$
R_{q}=\sqrt{\frac{1}{L} \int_{0}^{L} f^{2}(x) d x}
$$

Donde $R q$ es el cuadrado la distancia comprendida entre la línea de muestreo y la línea central que 
representa la deflexión del promedio de la raíz cuadrada obtenida al dividir por la longitud del avance transversal el área.

En la Figura 9 mediante la cámara de alta resolución es posible inspeccionar el cambio de la huella de contacto de la leva anular en las dos caras al finalizar cada período de prueba.

\section{Análisis digital de imágenes}

El análisis de la superficie de la leva anular de la bomba HP3 está en función de determinar factores de fricción, niveles de desgaste, resistencia, fatiga y lubricación de la pieza metálica con el propósito de establecer la eficiencia del combustible y el desempeño en el funcionamiento del motor diésel [26].

Para el análisis de las imágenes digitales [27], la interfaz gráfica en Matlab incorpora los algoritmos de registros de píxeles y subpíxeles de una secuencia de imágenes de baja resolución de la superficie mecánica para proceder a la binarización [28], luego las imágenes son filtradas aplicando un algoritmo de recuperación de fase para mejorar la identificación de resoluciones en función de los perfiles de relieve de la imagen [29], aplicando el teorema de convolución y la transformada inversa de Fourier se reconstruye la imagen resultante que muestra posibles zonas de mayor desgaste de la pieza mecánica [30]. Los principios matemáticos considerados en [31] son:

Transformada continua unidimensional de Fourier está dado por:

$$
F(u)=\int_{-\infty}^{\infty} f(x) \cdot e^{-j 2 \pi u x} d x
$$

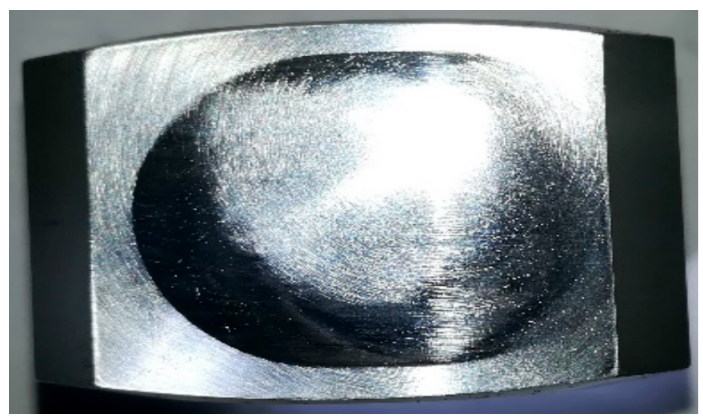

Figura 9. Inspección de huella de contacto de la leva anular.
Donde:

$F(u)$ es la función en el dominio de la frecuencia.

La transformada inversa unidimensional de Fourier está dada por:

$$
f(x)=\int_{-\infty}^{\infty} F(u) \cdot e^{-j 2 \pi u x} d u
$$

La amplitud o espectro de la onda está dado por:

$$
|F(u)|=\sqrt{R^{2}(u)+I^{2}(u)}
$$

Donde:

- $\quad R 2(u)$ es la parte real de la transformada de Fourier,

- $\quad$ I2(u) es la parte imaginaria de la transformada de Fourier.

Se considera el espectro de energía de la señal $f(x)$ dada por $|\boldsymbol{F}(\boldsymbol{u})| \mathbf{2}$

La fase de la onda está dado por

$$
\varnothing(u)=\operatorname{arctg}\left[\frac{I(u)}{R(u)}\right]
$$

El análisis del perfil de ondulación de la imagen está dado al implementar las ecuaciones desde la (3) hasta la (6) siendo estas ondas de aproximación al perfil unidimensional.

Para el tratamiento de la imagen es necesario extender la transformada de Fourier a su forma bidimensional, la misma que está dado por:

$$
F(u, v)=\int_{-\infty}^{\infty} \int_{-\infty}^{\infty} f(x, y) \cdot e^{j 2 \pi(u x+v y)} d x d y
$$

Donde:

$u y v$, representan las variables de frecuencia.

La transformada inversa bidimensional está dado por:

$$
f(x, y)=\int_{-\infty}^{\infty} \int_{-\infty}^{\infty} F(u, v) \cdot e^{-j 2 \pi(u x+v y)} d u d v
$$

Con la finalidad de analizar la discretización de la imagen digital se recurre a la transformada discreta de Fourier dada por: 
$F(u, v)=\frac{1}{M \cdot N} \sum_{x=0}^{M-1} \sum_{y=0}^{N-1} f(x, y) \cdot e^{-j 2 \pi\left(\frac{u x}{M}+\frac{v y}{N}\right)}(9)$

Donde:

- $\quad M$ es el número de columnas (píxeles en la dirección $x$ ), y

- $\quad N$ es el número de filas (píxeles en la dirección y).

La transformación inversa viene dada por la expresión:

$$
f(x, y)=\sum_{u=0}^{M-1} \sum_{v=0}^{N-1} F(u, v) \cdot e^{-j 2 \pi\left(\frac{u x}{M}+\frac{v y}{N}\right)}
$$

Con el algoritmo de la transformada discreta inversa de Fourier se consigue el componente de fase relevante en cuanto a la estructura espacial de la imagen [32]; además, contiene información sobre la posición relativa de las características o perfiles, siendo las zonas visibles con intensidad más elevada en la imagen [29] y para compensar la visualización del espectro se aplica la función.

$$
D(u, v)=c \cdot \log [1+|F(u, v)|]
$$

Donde:

$c$ es la constante de escalado.

Con la implementación de la función logarítmica se intenta realizar una compresión del rango dinámico, para facilitar la visualización e interpretación del espectro de Fourier.

En la ecuaciones desde la (7) hasta la (11) se implementan filtros con la intención de que el perfil de rugosidad este superpuesto al perfil de ondulación, en la Figura 10, se presenta la interfaz gráfica de la foto tomada a las 50 horas de funcionamiento, a la que se le denomina imagen original y el perfil mediante el análisis de Fourier se denomina imagen filtrada, de la cual se extrae los datos para su posterior análisis estadísticos y toma de decisiones.

En la Figura 11, está el análisis del espectro de potencia para las frecuencias verticales y horizontales de la representación matricial de la imagen utilizando la escala logarítmica dada en la ecuación (11).

La Figura 12, corresponde al histograma de la imagen filtrada que representa la cantidad de

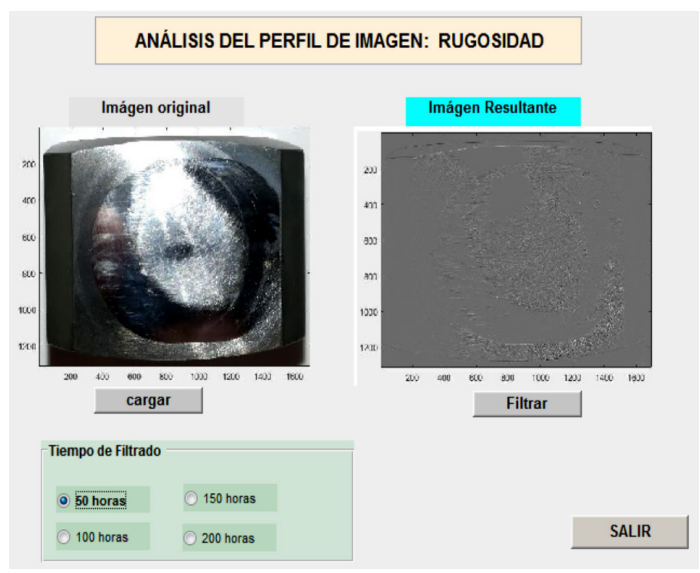

Figura 10. Interfaz gráfica para el análisis de la imagen.

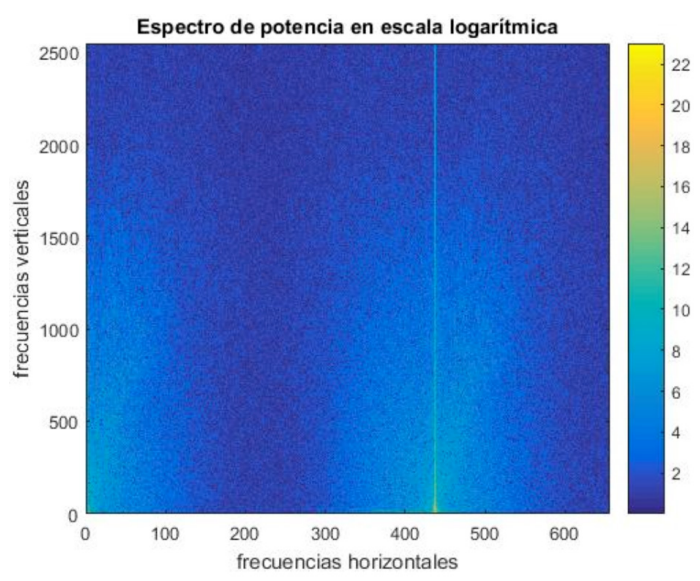

Figura 11. Espectro de potencia.

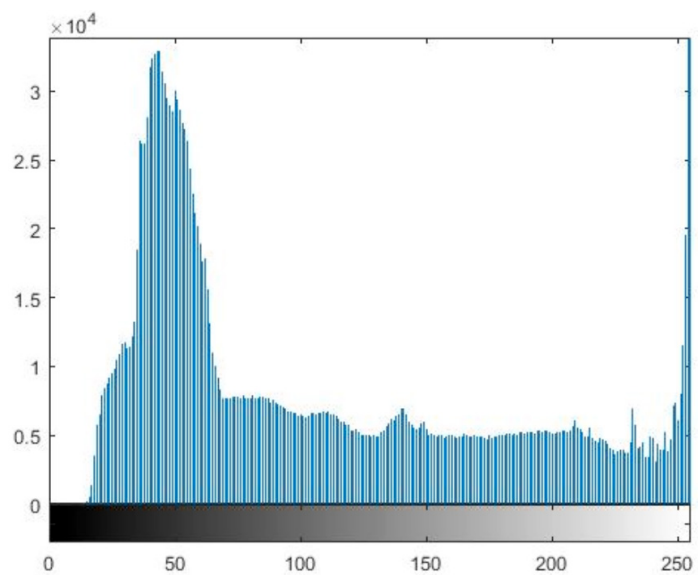

Figura 12. Histograma de intensidad de la imagen. 
pixeles con la misma intensidad en el eje vertical en función de la intensidad de 0 hasta 250 pixeles en el eje horizontal, los picos se interpreta como la abundancia de pixeles de una cierta intensidad dada por los anillos de Kapton [33].

\section{RESULTADOS}

Considerando [34] se detalla la estimación de los kilómetros recorridos por el vehículo en referencia a las horas de funcionamiento, generando las siguientes consideraciones:

- Régimen motor: $2500 \mathrm{rpm}$

- Relación caja: 4ta (1:1)

- Relación diferencial: 6,14

- Rin neumático: 17,5 in.

En la Figura 13 se representa el desgaste de la leva anular de la bomba, en donde se verifica la reducción de $0,121 \mathrm{~g}$ entre el valor máximo y mínimo que representa el $0,075 \%$ del peso inicial. El desgaste presentado es regular sin variaciones significativas. El punto de mayor desgaste se produjo entre 0 y 50 horas.

\section{Análisis de peso}

La Tabla 5 presenta los valores obtenidos del peso de la leva anular en la balanza analítica de precisión al finalizar cada período de pruebas, con la estimación de recorrido en kilómetros.

\section{Inspección visual}

El análisis visual de la leva anular, con la cámara profesional de alta resolución, permite revisar el

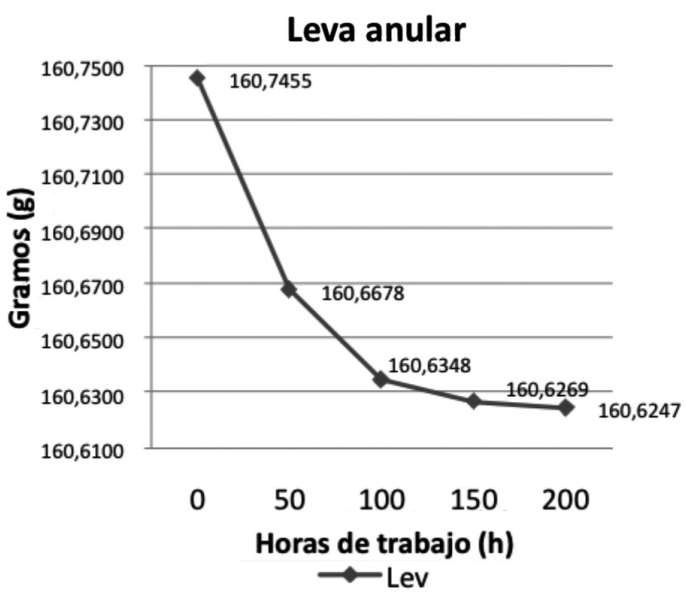

Figura 13. Desgaste de la leva anular.

estado del elemento, verificando el cambio de la huella de contacto en ambas caras, en 50, 100, 150 y 200 horas de trabajo con la mezcla diésel surfactante - agua, a una distancia y luz propuesta exacta en cada caso.

La Tabla 6, presenta el desgaste de la leva anular cara frontal de la bomba de alta presión, en cada período de prueba, la huella de contacto varía lo que indica una falta de lubricidad en la misma.

La Tabla 7 muestra el desgaste de la leva anular en la cara posterior de la bomba de alta presión en cada intervalo de funcionamiento, en donde se corrobora que la huella de contacto varía en cada período de

Tabla 5. Desgaste de la leva anular.

\begin{tabular}{|l|c|c|c|c|c|c|}
\hline \multirow{2}{*}{ Elemento } & 0 & 50 & 100 & 150 & 200 & Horas \\
& 0 & 3400 & 7000 & 10200 & 13600 & $\mathrm{Km}$ \\
\hline Leva anular & 160.7455 & 160.6678 & 160.6348 & 160.6269 & 160.6247 & Masa $(\mathrm{g})$ \\
\hline
\end{tabular}

Tabla 6. Comportamiento del material en la leva anular cara frontal.

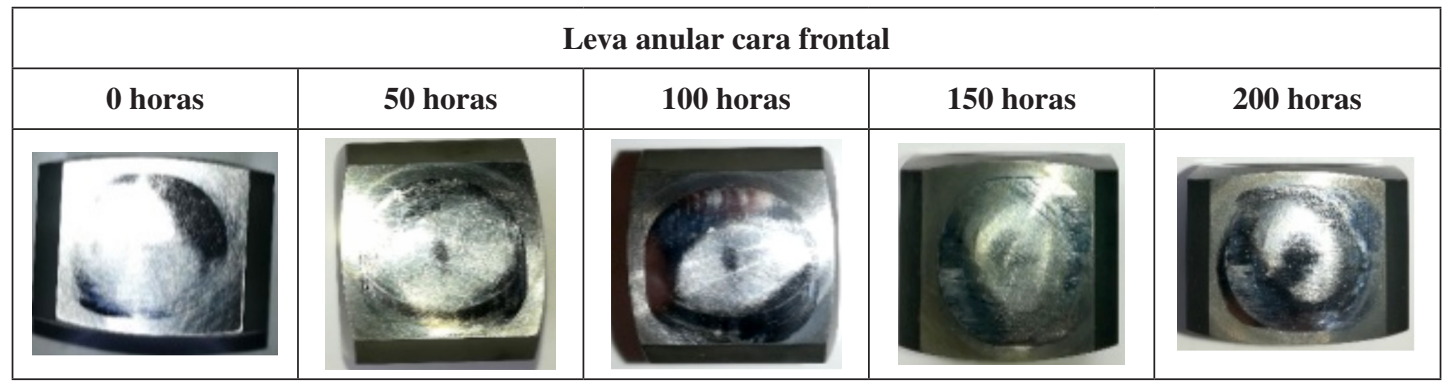


funcionamiento lo que indica una falta de lubricidad en la misma, al igual que en la cara frontal.

\section{Análisis de rugosidad}

[35] Para el análisis de rugosidad se considera el modelo matemático de análisis de regresión para proyectar el desgaste a futuro.

El análisis determinó las variaciones de rugosidad de la leva anular en ciclos de 50, 100, 150 y 200 horas de funcionamiento con la mezcla diéselsurfactantes - agua al $20 \%$.

\section{Cara frontal}

La Tabla 8 presenta los valores obtenidos con el rugosímetro al realizar el análisis a la leva anular en su cara frontal al finalizar cada protocolo de prueba.

En la Figura 14 se observa que la rugosidad aumenta conforme se suman las horas de funcionamiento, se obtiene una variación de $0,161 \mu \mathrm{m}$ entre el valor mínimo y máximo, aumentando su rugosidad en $22,33 \%$ con un máximo de $0,882 \mu \mathrm{m}$. Con el punto más crítico entre 50 y 100 horas.

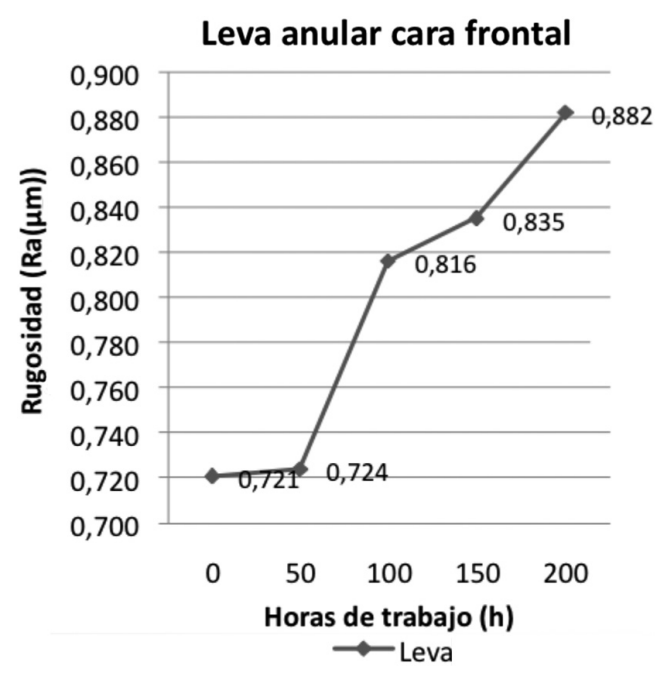

Figura 14. Valores de rugosidad en la leva anular cara frontal.

\section{Cara posterior}

En la Tabla 9 están los valores obtenidos con el rugosímetro al realizar el análisis a la leva anular en su cara posterior al finalizar cada protocolo de pruebas.

Tabla 7. Comportamiento del material en la leva anular cara posterior.

\begin{tabular}{|c|c|c|c|c|}
\hline \multicolumn{5}{|c|}{ Leva anular cara posterior } \\
\hline 0 horas & $\mathbf{5 0}$ horas & $\mathbf{1 0 0}$ horas & $\mathbf{1 5 0 \text { horas }}$ & 200 horas \\
\hline & & & & \\
\hline & & & & \\
\hline
\end{tabular}

Tabla 8. Valores de rugosidad en la leva anular cara frontal.

\begin{tabular}{|l|l|c|c|c|c|}
\hline \multicolumn{7}{|c|}{ Rugosidad leva anular cara frontal } \\
\hline Horas & 0 & 50 & 100 & 150 & 200 \\
\hline $\operatorname{Ra}(\mu \mathrm{m})$ & 0,721 & 0,724 & 0,816 & 0,835 & 0,882 \\
\hline
\end{tabular}

Tabla 9. Valores de rugosidad en la leva anular cara posterior.

\begin{tabular}{|l|l|c|c|c|c|}
\hline \multicolumn{7}{|c|}{ Leva anular cara posterior } \\
\hline Horas & 0 & 50 & 100 & 150 & 200 \\
\hline $\mathrm{Ra}(\mu \mathrm{m})$ & 0,560 & 0,572 & 0,701 & 0,749 & 0,786 \\
\hline
\end{tabular}


En la Figura 15 se representa el cambio de rugosidad que sufrió la superficie de contacto de la leva anular cara posterior, se observa que la medida de rugosidad aumentó conforme el paso de las horas de funcionamiento, se obtuvo una variación de $0,226 \mu \mathrm{m}$ entre el valor mínimo y máximo, con un creciente de su rugosidad en 40,35\% con un máximo de $0,786 \mathrm{z} \mu \mathrm{m}$. Con el punto más crítico entre 50 y 100 horas.

De la Tabla 8 los valores de rugosidad medidos de la leva anular en su cara frontal al finalizar las 200 horas, indican que el grado de rugosidad de su superficie es N6 [24], lo que indica que la huella de mecanización no es apreciable a simple vista.

En la cara posterior a diferencia de las medidas obtenidas de rugosidad en la cara frontal se observa que a las 0 y 50 horas de trabajo las medidas de $\mathrm{Ra}$ de $0,5 \mu \mathrm{m}$ que equivale a un grado de rugosidad de N5 lo cual indica que las marcas de mecanizado no se aprecian ni con el tacto ni con la vista pero si con lupa.

De acuerdo a la norma DIN 4768 [24], el obtener un grado de rugosidad N5 en el parámetro $R a$ implica que no sea perceptible para la visión humana, pero la deformación superficial en la leva anular, provoca la pérdida de la tolerancia que existe entre la leva anular y los émbolos buzos, lo que se traduce en un menor desplazamiento del mismo y a su vez una

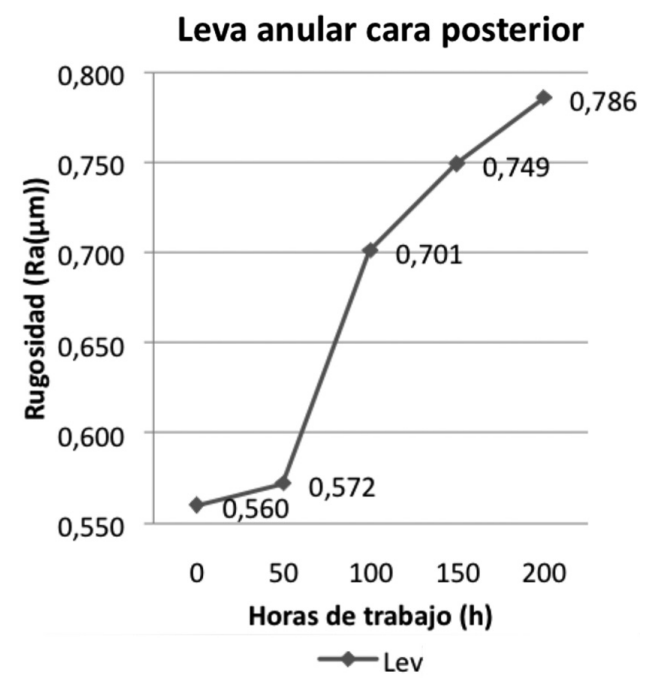

Figura 15. Valores de rugosidad en la leva anular cara posterior. disminución de la presión generada, provocando problemas en el rendimiento del motor, por lo que se justifica aplicar el análisis de Fourier para en análisis mediante el procesamiento de imágenes de las fotos digitales de la leva anular de la bomba HP3 DENSO tomadas a las 50 horas.

En la Figura 16, se presenta el resultado del comportamiento del perfil de la imagen de la cara frontal analizada a las 50 horas, el nivel de rugosidad de acuerdo a los algoritmos implementados en el análisis de Fourier es de 1,1 $\mu \mathrm{m}$ aproximadamente, generando una diferencia con el valor de $0,779 \mu \mathrm{m}$ dado por el rugosímetro, este valor marca una diferencia de 0,321 e indica que la lectura del rugosímetro es correcta y que no existió la presencia de factores externos que afecten la medición del nivel de rugosidad.

La prueba de hipótesis realizada en el software SPSS, verifica la incidencia del desgaste superficial de la leva en función del tipo de combustible que se utiliza, en la Tabla 10 se observa que existe una diferencia entre las medias de los perfiles de rugosidad a las

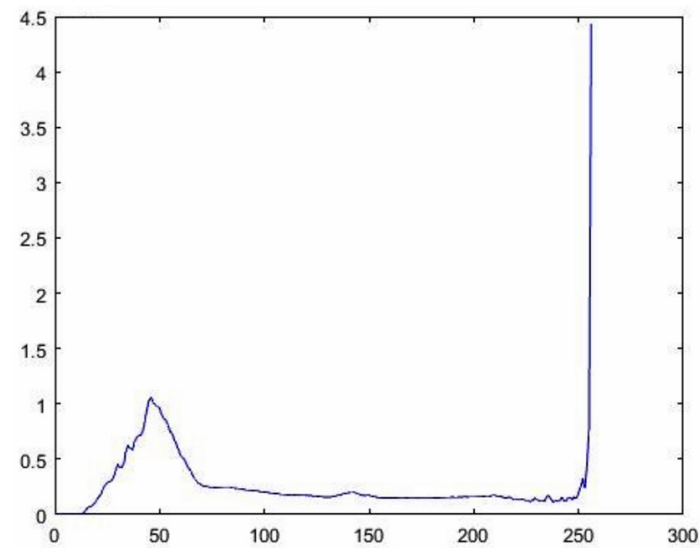

Figura 16. Valores de rugosidad en la leva anular cara posterior.

Tabla 10. Estadística descriptiva de la rugosidad entre dos levas.

\begin{tabular}{|l|c|c|c|}
\hline & Media & $\begin{array}{c}\text { Desviación } \\
\text { típica }\end{array}$ & $\begin{array}{c}\text { Error } \\
\text { típico de } \\
\text { la media }\end{array}$ \\
\hline $\begin{array}{l}\text { Diésel agua } \\
\text { - surfactante }\end{array}$ & 0,771664 & 0,2441410 & 0,0140955 \\
\hline Diésel & 0,597713 & 0,2923569 & 0,0168792 \\
\hline
\end{tabular}


50 horas entre la utilización del diésel y la mezcla ecológica combustible.

Para validar si la diferencia de medias es significativa, se aplica la prueba de hipótesis para muestras independientes y sus resultados se presentan en la Tabla 11 donde la prueba de Levence da un p-valor (Sig.) de 0,000 lo que indica que las varianzas no son iguales entre los dos grupos de análisis, luego el Sig. (bilateral) tiene un valor de 0,000 lo que se interpreta como la presencia significativa entre la media de rugosidad al utilizar el diésel y el diéselsurfactante, la misma se presenta en la Figura 17.

En la Figura 17 están los niveles de rugosidad producidas al utilizar el combustible ecológico diésel surfactante y el diésel como combustible entregan valores diferentes, al determinar un intervalo de confianza al 95\% para el parámetro $R a$.

[19] Como complemento y factor innovador de aporte investigativo, a la aplicación de la transformada de Fourier se incorpora la técnica de medición e investigación sobre los resultados de desempeño del

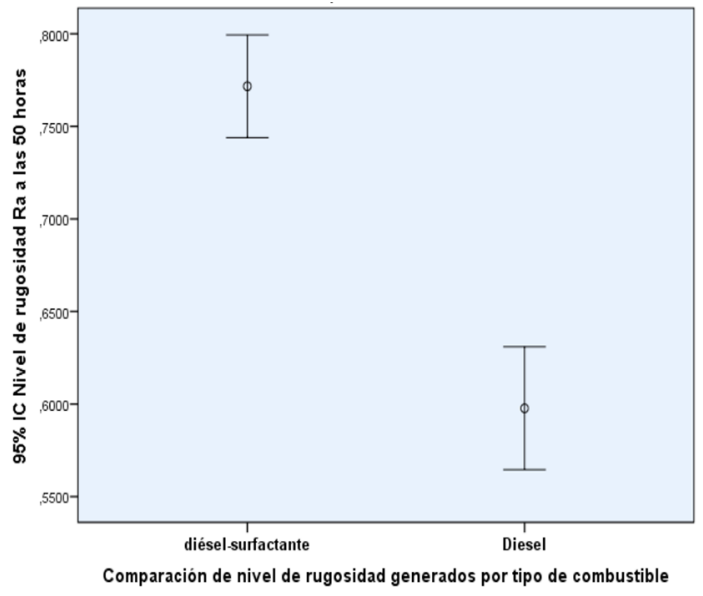

Figura 17. Comparación de niveles de rugosidad. motor a diésel en el dinamómetro al utilizar este combustible alternativo (biocombustible).

[36] Con el uso de combustibles emulsionados alternativos se eleva el rendimiento térmico y que en proporciones del $20 \%$ mejoran el desempeño mecánico (torque y potencia), reducen las emisiones y el poder calorífico en proporción al agua añadida.

[37] El combustible emulsionado que utiliza agua surfactante en la microemulsión de combustible, en un motor diésel de inyección directa, las emisiones de NOx decrecen con el incremento de agua en la formulación y reducen la opacidad de los gases de combustión.

La adición de agua en forma emulsión en el combustible tiene efectos positivos en la eficiencia de la combustión. Generan la elevación en el torque Figura 18 con el motor a velocidad constante de 1500 rpm en comparación a los resultados del diésel sin aditivo, porque se producen micro explosiones por la diferencia de volatilidad del agua. El agua se evapora en la cámara de combustión elevando las presiones del vapor contribuyendo a generar mayor torque efectivo del motor.

La potencia Figura 19, presenta mejores características a velocidad constante.

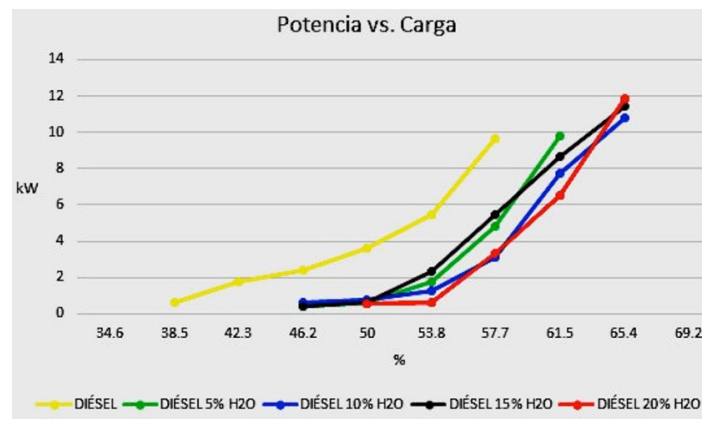

Figura 18. Incremento del torque con mezcla combustible.

Tabla 11. Prueba de muestras independientes.

\begin{tabular}{|l|c|c|c|c|c|}
\cline { 2 - 6 } \multicolumn{1}{c|}{} & \multicolumn{2}{c|}{$\begin{array}{c}\text { Prueba de Levence } \\
\text { (igualdad de varianzas) }\end{array}$} & \multicolumn{2}{c|}{ Prueba T para la igualdad de medias } \\
\hline & $\mathrm{F}$ & Sig. & $\mathrm{T}$ & $\mathrm{gl}$ & Sig. (bilateral) \\
\hline Asumido varianzas iguales & 14.715 & 0.000 & 7.910 & 598 & 0.000 \\
\hline Sin asumir varianzas iguales & & & 7.910 & 279.574 & 0.000 \\
\hline
\end{tabular}


El consumo específico de combustible aumenta para todas las emulsiones debido a la viscosidad que afecta en el patrón de pulverización de combustibley por la mayor densidad de la emulsión diésel.

El consumo específico de combustible Figura 20, es mayor en comparación al diésel a pesar de que las emulsiones por el incremento de potencia generada por el motor de combustión interna.

La emulsión de combustible disminuye el NOx, Figura 21 generadas por el motor en todos los

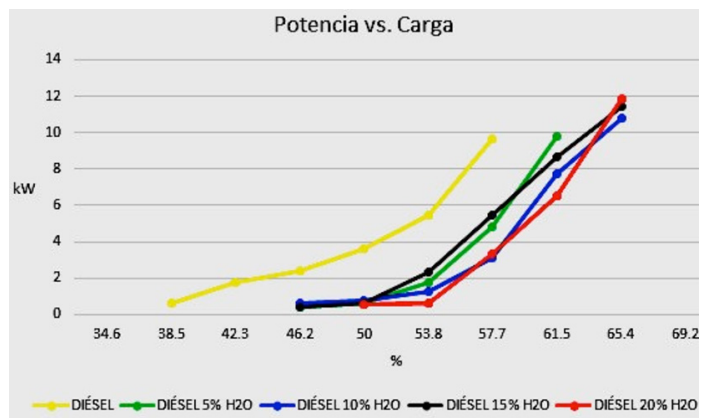

Figura 19. Incremento de potencia con mezcla combustible.

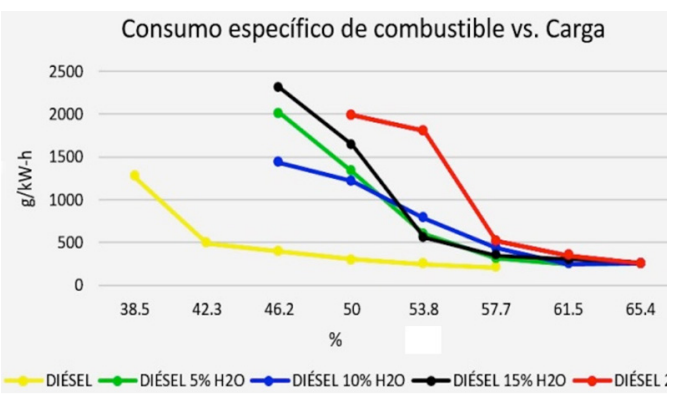

Figura 20. Consumo específico de combustible.

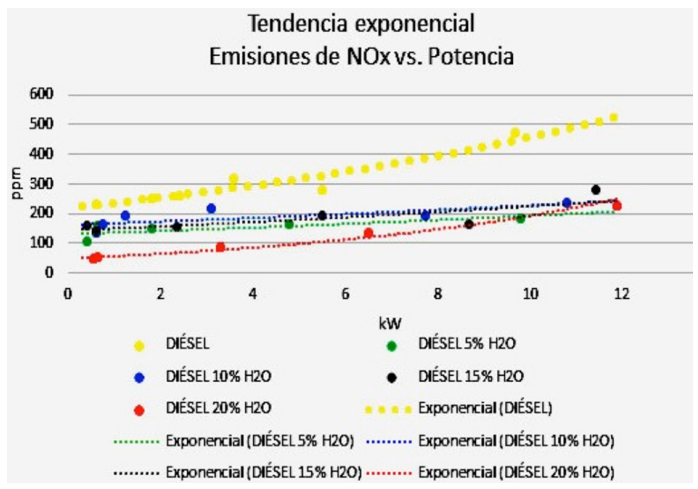

Figura 21. Emisiones de óxido nitroso NOx. rangos de potencia realizados en la prueba, siendo la emulsión con el $20 \%$ de agua la que presenta mejores resultados.

\section{CONCLUSIONES}

En el análisis de rugosidad de la leva anular en su cara frontal, tiene una variación de $0,161 \mu \mathrm{m}$, aumentando su rugosidad en $22,33 \%$.

En la cara posterior finaliza las pruebas, con un valor de $0,226 \mu \mathrm{m}$, aumentando su rugosidad en $40.35 \%$.

La masa de la leva anular se reduce en $0,121 \mathrm{~g}$ al finalizar el período de pruebas, valor que representa el $0,075 \%$ del peso inicial, lo que indica una falta de lubricidad en la misma.

La mezcla combustible a una concentración del $20 \%$ genera beneficios en la reducción de gases contaminantes como óxidos nitrosos y monóxido de carbono, sin embargo la presencia de agua y químicos generan desgaste prematuros en los componentes de la bomba de alta presión HP3 por la falta de lubricidad.

La interfaz gráfica en Matlab genera inconvenientes en el análisis de las propiedades geométricas de la imagen en términos de sus frecuencias, en especial al intentar definir una línea de referencia que sea validada como el parámetro $R a$, se utilizó filtros para excluir la ondulación y definir la rugosidad.

Según la norma DIN 4768, basados en la rugosidad de la leva anular se obtuvo un valor de $0,786 \mu \mathrm{m}$ en la cara posterior con grado $\mathrm{N} 5 \mathrm{y}$ un valor de $0.882 \mu \mathrm{m}$ en la cara frontal que pertenece al grado N6.

El análisis de rugosidad y de desgaste determina que el tiempo de vida útil de los componentes mecánicos que se encuentran en contacto con el combustible disminuye no así las propiedades de desempeño mecánico.

Las emulsiones de combustible al 20\%, disminuyen las emisiones de NOx al 50\% en comparación con el diésel lo que permite un equilibrio en la combustión.

El torque máximo alcanzado es para la emulsión con el $20 \%$ de agua - tensoactivos, en un similar del $20 \%$ que proporciona el diésel. 
La potencia máxima que se alcanza en esta prueba es para la emulsión al $20 \%$ de agua supera en un $18,5 \%$ al generado por el combustible diésel.

El consumo específico de combustible es mayor al usar emulsiones de agua y diésel siendo el más alto para la emulsión con el $15 \%$ de emulsión correspondiente a $261 \mathrm{~g} / \mathrm{kw}-\mathrm{h}$ superando por $55 \mathrm{~g} /$ kW-h al del diésel neto.

El procesamiento de imágenes y análisis desarrollado en este análisis es aplicable a componentes mecánicos que se someten a fricción y a cambios de rugosidad.

\section{REFERENCIAS}

[1] Denso Corporation. "Manual de servicio". Japón: Denso Corporation. 2004.

[2] E. Alptekin. "Evaluation of ethanol and isopropanol as additives with diesel fuel in a CRDI diesel engine. Fuel, pp. 161-172. 2017.

[3] V.W. Khond, and V.M. Kriplani. "Effect of nanofluid additives on performances and emissions of emulsified diesel and biodiesel fueled stationary CI engine: A comprehensive review". Renewable and Sustainable Energy Reviews, pp. 1338-1348. 2016.

[4] Ahmad et al., M. "Emulsifier-free Water-inDiesel emulsion fuel: Its stability behaviour, engine performance and exhaust emission". Fuel, pp. 454-462. 2018.

[5] K. Holmberg, B. Jönsson, B. Kronberg and B. Lindman. "Surfactants and polymers in aqueous solution". Inglaterra: John Wiley \& Sons, Ltd. 2003.

[6] Eliezer, A. (s.f.). "Caracterización del comportamiento de un motor mono-cilíndrico de encendido por compresión usando combustible emulsionado". Tesis de maestría. Instituto Superior Politécnico "José Antonio Echeverría”. La Habana .

[7] S. Sivakumar, B. Raju, and K. Rajulapati. Influence of $\mathrm{ZrB} 2$ hard ceramic reinforcement on mechanical and wear properties of aluminum. Ceramics International, pp. 1-54. 2018.

[8] A. Kotia, S. Borkakoti, and S. Kumar. "Wear and performance analysis of a 4-stroke diesel engine employing nanolubricants". Particuology, pp. 1-10. 2017.
[9] M. Hiroshi, and H. Alan. "Wear equation for adhesive wear established through elementary process of wear". Wear, pp. 186-192. 2013.

[10] Z. Xian-man, and C. Wei-ping. "Review on corrosion-wear resistance performance of materials in molten aluminum and its alloys". Transactions of Nonferrous Metals Society of China, pp. 1715-1731. 2015.

[11] G. Ospina, Y. Mohamed, A. Salah, I.H. Mohamed, and M.H. Adel. "Engine roughness and exhaust emissions of a diesel engine fueled with three biofuels". Renewable Energy, pp. 1-8. 2018.

[12] F. Xian-guang, W. Xiao-dong, C. Yu-xin, and W. Xin. "Image processing for three defects of topography images by SPM". Chemometrics and Intelligent Laboratory Systems, 185, pp. 12-17. 2019. https://doi. org/10.1016/j.chemolab.2018.12.013

[13] K. Uruma, K. Konishi, T. Takahashi, and T. Furukawa. "Colorization-based image coding using graph Fourier transform". Signal Processing: Image Communication. 2018. URL: https://doi.org/10.1016/j. image.2018.12.011

[14] X. Su, R. Tao and X. Kang. "Analysis and Comparison of Discrete Fractional Fourier Transforms". Signal Processing. 2019. URL: https://doi.org/10.1016/j.image.2018.12.011

[15] M.H. Annaby, H.A. Ayad, M.A. Rushdi, and E.A. Nehary. "Difference operators and generalized discrete fractional transforms in signal and image processing". Signal Processing, 151, pp. 1-18. 2018. URL: https:// doi.org/10.1016/j.sigpro.2018.04.023

[16] A. Rashid, and O. Hasan. "Formal analysis of continuous-time systems using Fourier transform". Journal of Symbolic Computation, 90, pp. 65-88. 2019. URL: https://doi. org/10.1016/j.jsc.2018.04.004

[17] C. Ma, H. Lin, G. Zhang, and R. Du. "Digital micro-mirror device based multispectral imaging using compressed Fourier spectrum". Optics Communications, 426, pp. 348358. 2018. URL: https://doi.org/10.1016/j. optcom.2018.05.067

[18] D.O. Dolmatov, D.G. Demyanyuk, A.H. Ozdiev, and R.V. Pinchuk. "Fourier-domain post-processing technique for Digital Focus Array imaging with Matrix phased array for ultrasonic testing of ITER components". 
Fusion Engineering and Design, 126, 124129. 2018. URL: https://doi.org/10.1016/j. fusengdes.2017.11.020

[19] D. Pucuji, and G. Erazo. "Investigación del potencial energético de la emulsión diesél -agua al 5-10-15 Y 20\% con el uso de surfactante para determinar su influencia en los parámetros mecánicos y térmicos del motor de combustion interna". Tesis de Pregrado. Universidad de las Fuerzas Armadas ESPE, Latacunga. 2016.

[20] H. Carrillo, C. Taco, and G. Erazo. "Análisis termográfico y control de emisiones de la mezcla combustible diésel - surfactantes del agua en el motor MZR-D 2.5". Tesis de pregrado. Universidad de las Fuerzas Armadas ESPE, Latacunga. 2018.

[21] C. Salinas, and R. Villavicencio. "Diseño y construcción mecánica de un banco de comprobación de inyectores diesel: Denso, Delphi, Bosch del sistema CRDI". Tesis de pregrado. Univerdidad de las Fuerzas Armadas ESPE, Latacunga. 2013.

[22] Z. Xin, Y.ZhangYi' an, D. Xiaochuan, L. Xuan, Q. Ming, L. Zhaoji, and Z. Bo. "Investigation on $4 \mathrm{H}-\mathrm{SiC}$ MOSFET with Three-Section Edge Termination". Superlattices and Microstructures. 2018.

[23] R. Analuisa, and E. Vizcaino. "Análisis de durabilidad de los materiales de fabricación del inyector denso 6521 del sistema de inyección crdi al trabajar con la mezcla combustible obtenido a partir del aceite de motor reciclado". Tesis de Pregrado. Universidad de las Fuerzas Armadas ESPE, Latacunga. 2016.

[24] B. Brodnik Zugelj, and M. Kalin. "Submicronscale experimental and theoretical analyses of multi-asperity contacts with different roughnesses”. Tribology International. 2018.

[25] Y. Su, Q. Zhang, Z. Fang, Y. Wang, Y. Liu, and $\mathrm{S}$. Wu. "Elimination of systematic error in digital image correlation caused by intensity interpolation by introducing position randomness to subset points". Optics and Lasers in Engineering. Vol. 114, pp. 60-75. 2019. URL: https://doi.org/10.1016/j. optlaseng.2018.10.012

[26] L.F. Castro. "Rugosímetros: herramientas sencillas para grandes resultados". Revista metal actual. Vol. 16, pp. 5-9. 2010.
[27] R. Horstmeyer, X. Ou, G. Zheng, P. Willems, and C. Yang. "Digital pathology with Fourier ptychography". Computerized Medical Imaging and Graphics. Vol. 42, pp. 38-43. 2015. URL: https://doi.org/10.1016/j. compmedimag.2014.11.005

[28] M.J. Sun, J.Y. Huang, and J. Penuelas. "Suppressing the noise in binarized Fourier single-pixel imaging utilizing defocus blur". Optics and Lasers in Engineering. Vol. 108, pp. 15-18. 2018. URL: https://doi. org/10.1016/j.optlaseng.2018.04.013

[29] C. Wang, X. Wang, Y. Li, Z. Xia, and C. Zhang. "Quaternion polar harmonic Fourier moments for color images". Information Sciences. Vol. 450, pp. 141-156. 2018. URL: https://doi.org/10.1016/j.ins.2018.03.040

[30] A. Torres, C.J. Luis, and I. Puertas. "Spacing roughness parameters analysis on the EDM of TiB2”. Procedia Manufacturing. Vol. 13, pp. 579-584. 2017.

[31] L.Á. Ruiz Fernández. La transformada de Fourier. Aplicación al filtrado de imágenes. 2016.

[32] X. Wu, and X. Yin. "Surface Roughness Analysis and Parameter Optimization of Mold Steel Milling". Procedia CIRP, 71, pp. 317321. 2018. URL: https://doi.org/10.1016/j. procir.2018.05.032

[33] N. Triana, A.E. Jaramillo, R.M. Gutiérrez, and C.A. Rodríguez. "Técnicas de umbralización para el procesamiento digital de imágenes de GEM-Foils". Scientia et technica. Vol. 21 $\mathrm{N}^{\circ}$ 4, pp. 352-359. 2016.

[34] J. Bassante, S. Díaz, and G. Erazo. "Análisis del desgaste del pulverizador y comportamiento electrónico de inyectores crdi y bomba de alta presión cp al utilizar la mezcla combustible diesel surfactantes de agua". Tesis de pregrado. Universidad de las Fuerzas Armadas ESPE, Latacunga. 2019.

[35] X. Weiwei, Z. Konghao, W. Jianjun, L. Yajun, and L. Qiang. "Modeling and numerical analysis of the effect of blade roughness on particle deposition in a flue gas turbine". Powder Technology. 2019. URL: https://doi. org/10.1016/j.powtec.2019.02.033

[36] T. Kannan, and R. Marappan. "Thevetia peruviana biodiesel emulsion used as a fuel in a single cylinder diesel engine reduces NOx 
and smoke". Thermal Science, pp. 11851191. 2011.

[37] A. Dantas, M. Fernandes, E. Barros, T. Castro, and M. Moura. "Alternative fuels composed by blends of nonionic surfactant with diesel and water: engine performance and emissions". Brazilian Journal of Chemical Engineering, pp. 521-531. 2011. 